\title{
CORRIGENDUM
}

doi:10.1038/nature 16488

\section{Corrigendum: Dinosaurian growth patterns and rapid avian growth rates}

Gregory M. Erickson, Kristina Curry Rogers \& Scott A. Yerby

\section{Nature 412, 429-433 (2001); doi:10.1038/35086558}

Questions have been raised about the methods used in the construction of dinosaurian growth curves in this Letter ${ }^{1}$. These were caused by ambiguity with regard to how curve-fitting functions were utilized, and insufficient explanation for how maximum growth rates were calculated. Taken together, these omissions gave the impression that we were able to fit very specific curves even in cases where data were seemingly too scarce to justify them. We apologise for the confusion. However, the main conclusions of the paper were not affected. A detailed rationale is available in the Supplementary Methods and Supplementary Discussion of this Corrigendum and the source data are provided as Supplementary Data. We thank N. Myhrvold for bringing these issues to our attention.

In our reanalysis we found the following translational mistakes, which do not appear to have contributed to Myhrvold's concerns; however, we take this opportunity to rectify them. The growth rates for Psittacosaurus mongoliensis were incorrectly reported as $5.82 \mathrm{~kg} \mathrm{yr}^{-1}$ versus $5.28 \mathrm{~kg} \mathrm{yr}^{-1}$ in Fig. 2 and $12.5 \mathrm{~g} \mathrm{~d}^{-1}$ in the legend to Fig. 3. Fortunately, the correct value of $14.1 \mathrm{~g} \mathrm{~d}^{-1}$ was used in the comparative regression calculations. Finally, the mass estimate used for one of the Apatosaurus specimens was incorrectly transcribed. This modestly affected the growth curve parameters in Fig. 2. Details can be found in the Supplementary Methods and Discussion to this Corrigendum along with the corrected Fig. 2. The change causes a negligible shift in the overall dinosaur regression line slope (see the Supplementary Data to this Corrigendum) and does not compromise our conclusion that dinosaurs grew like endotherms.

Supplementary Information is available in the online version of the Corrigendum.

1. Myhrvold, N. P. Revisiting the estimation of dinosaur growth rates. PLOS ONE 8, http://dx.doi.org/10.1371/journal.pone.0081917 (2013). 